\title{
Multiplicity scaling in ideal and viscous hydrodynamics
}

\author{
Huichao Song ${ }^{1, *}$ and Ulrich Heinz ${ }^{1,2}$ \\ ${ }^{1}$ Department of Physics, Ohio State University, Columbus, OH 43210, USA \\ ${ }^{2}$ CERN, Physics Department, Theory Division, $\mathrm{CH}-1211$ Geneva 23, Switzerland
}

(Received 12 May 2008; published 12 August 2008)

\begin{abstract}
Using numerical results from ideal and viscous relativistic hydrodynamic simulations with three different equations of state, for $\mathrm{Au}+\mathrm{Au}$ and $\mathrm{Cu}+\mathrm{Cu}$ collisions at different centralities and initial energy densities, we explore the dependence of the eccentricity-scaled elliptic flow, $v_{2} / \varepsilon$, and the produced entropy fraction, $\Delta \mathcal{S} / \mathcal{S}_{0}$, on the final charged hadron multiplicity density $d N_{\mathrm{ch}} / d y$ per unit transverse overlap area $S,(1 / S) d N_{\mathrm{ch}} / d y$. The viscous hydrodynamic simulations are performed with two different versions of the Israel-Stewart kinetic evolution equations, and in each case we investigate the dependence of the physical observables on the kinetic relaxation time. We find approximate scaling of $v_{2} / \varepsilon$ and $\Delta S / S_{0}$ with $(1 / S) d N_{\mathrm{ch}} / d y$, with scaling functions that depend on the EOS and, in particular, on the value of the specific shear viscosity $\eta / s$. Small scaling violations are seen even in ideal hydrodynamics, caused by a breaking of the scale invariance of ideal fluid dynamics by the freeze-out condition. Viscous hydrodynamics shows somewhat larger scale-breaking effects that increase with increasing $\eta / s$ and decreasing system size and initial energy density. We propose to use precision studies of these scaling violations to help constrain the shear viscosity $\eta / s$ of the quark-gluon plasma created in relativistic heavy ion collisions.
\end{abstract}

DOI: 10.1103/PhysRevC.78.024902

PACS number(s): 12.38.Mh, 24.10.Nz, 25.75.Ld

\section{INTRODUCTION}

Recent numerical studies of relativistic hydrodynamics for dissipative fluids [1-4] have confirmed earlier estimates $[5,6]$ that the "elliptic" anisotropic collective flow observed in noncentral heavy-ion collisions is very sensitive to the shear viscosity of the matter formed in such collisions. The fact that ideal fluid dynamics (i.e., the assumption that viscosity can be neglected) provides a phenomenologically quite successful description of much of the soft hadron data collected from $\mathrm{Au}+\mathrm{Au}$ collisions at the BNL Relativistic Heavy Ion Collider (RHIC) [7-9] implies that there are strong constraints on the shear viscosity to entropy ratio $\eta / s[6,10]$ and the thermalization time scale $[11,12]$ of the matter in the collision fireball. The conclusion is that the quark-gluon plasma (QGP) created at RHIC is a strongly coupled plasma with almost perfect liquid collective behavior [13-15] whose specific shear viscosity is lower than that of any previously known real fluid and consistent with a postulated lower bound of $\eta / s \geqslant \hbar /\left(4 \pi k_{B}\right)$ derived from the study of infinitely strongly coupled conformal field theories $[16,17]$ using the anti-de-Sitter space/conformal field theory (AdS/CFT) correspondence and corroborated by earlier quantum mechanical arguments based on the uncertainty relation [18].

On the other hand, heavy-ion data obtained from RHIC, CERN Super Proton Synchrotron (SPS), and BNL Alternating Gradient Synchrotron (AGS) also show that ideal hydrodynamics gradually breaks down at larger impact parameters, for smaller collision systems, at lower collision energies, and away from midrapidity (see Ref. [9] for a review). Much of this can be attributed to strong viscous effects in the late collision stage after the QGP has converted to hadrons [19]. However,

*song@mps.ohio-state.edu due to uncertainties in the initial conditions for the fireball deformation, there is some room left for a nonzero value of the QGP viscosity [19]. To study this further requires a viscous hydrodynamic approach because the tool used to describe the nonequilibrium late hadronic dynamics (a classical cascade) is not well suited for the rapidly evolving, very dense matter in the early collision stages.

The motivation for the present paper is provided by the well-known systematic comparison of Voloshin et al. [20,21] of elliptic flow data with ideal fluid dynamical predictions which suggests that the elliptic flow parameter $v_{2}$ scaled by the initial source eccentricity $\varepsilon, v_{2} / \varepsilon$, while strongly deviating from ideal hydrodynamics at low multiplicities, still scales with the final multiplicity per unit overlap area:

$$
\frac{v_{2}}{\varepsilon} \propto \frac{1}{S} \frac{d N_{\mathrm{ch}}}{d y} .
$$

For ideal fluids, the right-hand side is a direct measure of the initial entropy density [22]. The scaling in Eq. (1) implies that all dependence on impact parameter, collision energy, and system size can be, to good approximation, absorbed by simply taking into account how these control parameters change the final hadron multiplicity density. We will call this observation simply "multiplicity scaling of the elliptic flow," where "elliptic flow" is short for the eccentricity-scaled elliptic flow $v_{2} / \varepsilon$ and "multiplicity" stands for $(1 / S) d N_{\mathrm{ch}} / d y$.

Such a scaling is expected for ideal fluid dynamics whose equations of motion are scale invariant and where the eccentricity-scaled elliptic flow is therefore predicted $[23,24]$ to depend only on the squared speed of sound, $c_{s}^{2}=\frac{\partial p}{\partial e}$, which describes the stiffness of the equation of state (EOS) or "pushing power" of the hydrodynamically expanding matter. It has been known for many years [25], however, that this ideal-fluid scaling is broken by the final freeze-out of the matter: if hadron freeze-out is controlled 
by hadronic cross sections (mean free paths) or simply parametrized by a critical decoupling energy density $e_{\mathrm{dec}}$ or temperature $T_{\mathrm{dec}}$, this introduces an additional scale into the problem that is independent of (or at least not directly related to) the initial geometry of the fireball. This breaks the above argument based on scale invariance of the ideal fluid equations of motion. We will show here that this also leads to a breaking of the multiplicity scaling of $v_{2} / \varepsilon$ not only in the most peripheral or lowest energy collisions, where freeze-out obviously cuts the hydrodynamic evolution short since the freeze-out density is reached before the flow anisotropy can fully build up [25], but even in the most central collisions at RHIC where freeze-out still terminates the hydrodynamic evolution before the elliptic flow can fully saturate (see also Ref. [19]).

The more interesting aspect of the experimentally observed scaling is, however, its apparent validity in regions where ideal fluid dynamics does not work (these encompass most of the available data [20]). [For a recent discussion of possible origins of the observed multiplicity scaling see Ref. [26].] Many years ago, simple scaling laws for the centrality dependence of elliptic flow were derived from kinetic theory in the dilute gas limit, where the particles in the medium suffer at most one rescattering before decoupling [27,28]; these can be reinterpreted in terms of multiplicity scaling for $v_{2} / \varepsilon$. The dilute gas limit is expected to hold for very small collision systems, very large impact parameters or very low collision energies. More recently, a successful attempt was made to phenomenologically connect the dilute gas and hydrodynamic limits with a one-parameter fit involving the Knudsen number [29]. This fit works very well for $\mathrm{Au}+\mathrm{Au}$ and $\mathrm{Cu}+\mathrm{Cu}$ data from RHIC, but it predicts that even in the most central $\mathrm{Au}+\mathrm{Au}$ collisions at RHIC the ideal fluid dynamical limit has not yet been reached and is missed by at least $25 \%$ [29]. In the present paper we use viscous relativistic hydrodynamics to explore the multiplicity scaling of $v_{2} / \varepsilon$ in the phenomenologically relevant region. We conclude (not surprisingly since much of the available data is from regions where the viscous hadronic phase plays a large role [19]) that the multiplicity scaling data [20,21] require significant shear viscosity for the medium, but also that viscous hydrodynamics predicts subtle scaling violations that seem to be qualitatively consistent with trends seen in the data (even if the experimental evidence for scaling violations is presently not statistically robust) and whose magnitude is sensitive to the specific shear viscosity $\eta / s$. This gives us hope that future more precise data can help constrain the QGP shear viscosity through exactly such scaling violations.

We should caution the reader that, similar to Ref. [29] which used a constant (time-independent) Knudsen number, our viscous hydrodynamic calculations are done with a constant (temperature-independent) specific shear viscosity $\eta / s$. Neither assumption is realistic, and we expect $\eta / s$ in particular to show strong temperature dependence near $T_{\mathrm{c}}$ (the critical temperature for the quark-hadron phase transition) and emerge from the phase transition with much larger values than in the QGP phase. Comparisons between the results presented here and experimental data are therefore, at best, indicative of qualitative trends, and improved calculations, which in particular match viscous hydrodynamics to a realistic hadron cascade below $T_{\mathrm{c}}$, are required before an extraction of $\eta / s$ from experimental data can be attempted.

\section{DISSIPATIVE FLUID DYNAMICS}

In this section we briefly review the viscous hydrodynamic equations that we solve, focusing on some differences in the formulations used in previously published papers [1-4] which we investigate here further. For technical details we ask the reader to consult these earlier papers.

We focus on systems with exact longitudinal boost invariance and use the code VISH2+1 [3] to solve numerically for the expansion in the two dimensions transverse to the beam direction. As in Ref. [1-4] we consider only shear viscosity, neglecting bulk viscosity and heat conduction. (Bulk viscosity may become large near the QCD phase transition [30-32] and should thus be included in the future before comparing viscous hydrodynamics with experimental data.) VISH2+1 solves the conservation laws for energy and momentum, $d_{m} T^{m n}=0$ [where $d_{m}$ is the covariant derivative in our curvilinear ( $\tau, x, y, \eta)$ coordinate system [33]], with the decomposition

$T^{m n}=e u^{m} u^{n}-p \Delta^{m n}+\pi^{m n}, \quad \Delta^{m n}=g^{m n}-u^{m} u^{n}$,

together with the evolution equations for the viscous shear pressure tensor components $\pi^{m n}$ :

$$
\begin{aligned}
D \pi^{m n}= & -\frac{1}{\tau_{\pi}}\left(\pi^{m n}-2 \eta \sigma^{m n}\right)-\left(u^{m} \pi^{n k}+u^{n} \pi^{m k}\right) D u_{k} \\
& -\frac{1}{2} \pi^{m n} \frac{\eta T}{\tau_{\pi}} d_{k}\left(\frac{\tau_{\pi}}{\eta T} u^{k}\right)-\pi_{a}^{(m} \omega^{n) a} .
\end{aligned}
$$

Here $D=u^{m} d_{m}$ is the time derivative in the local comoving frame, $\nabla^{m}=\Delta^{m l} d_{l}$ is the spatial gradient in that frame, and $\sigma^{m n}=\nabla^{\langle m} u^{n\rangle}=\frac{1}{2}\left(\nabla^{m} u^{n}+\nabla^{n} u^{m}\right)-\frac{1}{3} \Delta^{m n} \theta$ (with the scalar expansion rate $\theta \equiv d_{k} u^{k}=\nabla_{k} u^{k}$ ) is the symmetric and traceless velocity shear tensor. $\omega_{m n}=\nabla_{n} u_{m}-\nabla_{m} u_{n}$ is the vorticity tensor, and $a^{(m} b^{n)} \equiv \frac{1}{2}\left(a^{m} b^{n}+a^{n} b^{m}\right)$ denotes symmetrization. Even though several components of the symmetric shear pressure tensor $\pi^{m n}$ are redundant [33] on account of its tracelessness and transversality to the flow velocity $u^{m}$, VISH2+1 propagates all 7 nonzero components and uses the tracelessness and transversality conditions as checks of the numerical accuracy [3].

For a conformally symmetric fluid such as a massless quarkgluon gas, the temperature $T$ is the only scale in the problem, and therefore $\eta \sim s \sim T^{3}$ and $\tau_{\pi} \sim 1 / T$, hence $\eta T / \tau_{\pi} \sim T^{5}$. In this limit, the first term in the second line of Eq. (3) can be written as [34]

$$
-\frac{1}{2} \pi^{m n} \frac{\eta T}{\tau_{\pi}} d_{k}\left(\frac{\tau_{\pi}}{\eta T} u^{k}\right)=+\frac{1}{2} \pi^{m n}(5 D(\ln T)-\theta) .
$$

This is the form used in Ref. [1].

It has recently been argued [35-38] that the right-hand side of Eq. (3) should contain even more terms, at least for conformal fluids in the strong coupling limit. We will not pursue this possibility here.

Equation (3) is known as the Israel-Stewart (I-S) equation and is based on an expansion of the entropy production rate to second order [34,39-42] (macroscopic approach) or, 
in a microscopic kinetic approach using Grad's 14-moment method, of the phase-space distribution function to first order in small deviations from local thermal equilibrium [39,42,43] (see also Ref. [44]). By introducing a finite and sufficiently large relaxation time $\tau_{\pi}$ for the evolution of the shear pressure tensor toward its Navier-Stokes limit $\pi^{m n}=2 \eta \sigma^{m n}$, this equation eliminates problems with acausal signal propagation at short wavelengths and the resulting numerical instabilities that famously plague the relativistic Navier-Stokes equation. A somewhat different approach to solving these problems was developed by Öttinger and Grmela (Ö-G) [45] and has been used in Ref. [4]; because a comparison of results obtained with the I-S and Ö-G equations is nontrivial, we will leave that for a later study.

References [1-3] use different versions of Eq. (3). Romatschke and Romatschke [1] use the full set of terms displayed in Eq. (3), which we label as the "full I-S equation." The last term in the second line of Eq. (3) involving the vorticity cannot be obtained from the macroscopic approach $[33,41]$ since it does not contribute to entropy production, but it follows from the microscopic kinetic approach [39,43]. For longitudinally boost-invariant systems with initially vanishing transverse flow, it is zero initially and was found in Ref. [1] to remain tiny throughout the fireball evolution. We can therefore remove it from consideration when comparing published results from the different approaches. The first term in the second line of Eq. (3) arises in this form from the macroscopic approach (second-order entropy production $[33,41]$ ) but was neglected in our previous work [3], following an argument in Ref. [33] that it is of second order in small quantities and therefore subdominant compared to the first two terms on the right-hand side of Eq. (3). A similar argument can be made for the last term in line 1 of Eq. (3) (which does not contribute to entropy production either), but Baier et al. [43] pointed out that this term is needed to preserve the transversality of $\pi^{m n}$ during kinetic evolution. In Ref. [3] we therefore kept all terms in the first line of Eq. (3) but dropped those in the second line; we call this here the "simplified I-S equation." In Ref. [35] Baier et al. argued that for a conformally invariant medium, such as a classical massless quark-gluon gas, the first term in the second line is needed to preserve the conformal invariance of the kinetic evolution equation and hence should not be dropped. We now understand that the arguments presented in Ref. [33] to neglect all but the first term on the right-hand side of Eq. (3) were at best superficial, since this term involves the difference between two first-order quantities and thus presumably needs to be counted as small of second order.

Chaudhuri followed in his work [2] the approach advertised in Ref. [33]; as a result, in his procedure, $\pi^{m n}$ must be expected to evolve away from transversality. He circumvents this problem by evolving only the three linearly independent components of $\pi^{m n}$ and computing the rest from the tracelessness and transversality conditions [2]. The problem resurfaces, however, since now the results for all components of $\pi^{m n}$ must be expected to depend on the choice of independent components which are evolved dynamically with the truncated Eq. (3). While these questions await quantitative study, we note that Chaudhuri's results [2] appear to differ significantly from ours [3].
In the present paper, we show many comparisons between solutions obtained by using the full I-S equation with those from the simplified I-S equation. Although at sufficiently long wavelengths both have to agree in the Navier-Stokes limit $\tau_{\pi} \rightarrow 0$ (up to issues of numerical stability), as inspection of Eq. (3) readily shows, they differ for nonzero $\tau_{\pi}$ and will be seen to exhibit different degrees of sensitivity to $\tau_{\pi}$. This is of phenomenological importance, since $\tau_{\pi}$ for the QGP is not known, and a strong sensitivity to this unknown parameter will negatively impact our ability to extract the QGP shear viscosity $\eta / s$ from experimental data.

\section{INITIAL CONDITIONS, FREEZE-OUT, AND EQUATION OF STATE}

For the present study, we initialize the expanding fireball in the same way as in Ref. [3], i.e., with vanishing initial transverse flow and with an initial energy density profile proportional to the transverse density of wounded nucleons, calculated from a Saxon-Woods nuclear density profile with radius and surface thickness parameters $R_{0}=4.2 \mathrm{fm}, \xi=$ $0.596 \mathrm{fm}$ for $\mathrm{Cu}$ and $R_{0}=6.37 \mathrm{fm}, \xi=0.56 \mathrm{fm}$ for $\mathrm{Au}$ nuclei. The energy density profile is normalized by a parameter $e_{0}=e\left(\tau_{0} ; r=b=0\right)$ giving the peak energy density in the center of the fireball for central collisions (impact parameter $b=0$ ). $e_{0}$ is related to the peak wounded nucleon density in the same collisions by a factor $\kappa$ which is assumed to depend on energy but not on the size of the colliding nuclei. We here consider $e_{0}$ values that lead to final multiplicities covering the range accessible at RHIC and beyond, albeit perhaps not all the way to the Large Hadron Collider (LHC).

As of now, the energy dependence of $\kappa$ cannot be calculated and must be determined empirically from the final charged hadron multiplicity $d N_{\mathrm{ch}} / d y$. Since $d N_{\mathrm{ch}} / d y$ counts the final entropy per unit of rapidity, including any entropy generated by viscous effects during the expansion, the value of $d N_{\mathrm{ch}} / d y$ corresponding to a given $\kappa$ will depend on the viscosity $\eta / s$. We will see that the amount of entropy produced by viscous effects additionally depends on system size, impact parameter, and collision energy, but that all these dependences can, to good approximation, be absorbed in a single scaling function, with parametric dependence on $\eta / s$, that depends only on the multiplicity density $(1 / S) d N_{\mathrm{ch}} / d y$ : similar to $v_{2} / \varepsilon$, entropy production $\Delta \mathcal{S} / \mathcal{S}_{0}$ exhibits approximate "multiplicity scaling." However, this scaling function turns out to be nonlinear. It therefore modifies the centrality dependence of the produced charged multiplicity, softening the observed increase with collision centrality of the produced charged multiplicity per pair of wounded nucleons, $2 \frac{d N_{\mathrm{ch}} / d y}{N_{\mathrm{part}}}$. Exploration of this important issue requires an accurate modeling of the impact parameter dependence of the initial entropy density profile using, say, the Glauber or color glass condensate models. This is beyond the scope of the present article and will be left for a future study.

Following the majority of previous studies [2-4,46,47], the viscous shear pressure tensor is initialized with its NavierStokes value $\pi^{m n}=2 \eta \sigma^{m n}$. Other initial conditions were studied in Refs. [1,3], but the final observables were found 
to be insensitive to such variations [3]. The kinetic relaxation time $\tau_{\pi}$ for the kinetic evolution of the shear pressure tensor is taken as $\tau_{\pi}=c_{\pi} \tau_{\pi}^{\text {Boltz }}$, where $\tau_{\pi}^{\text {Boltz }}=\frac{6}{T} \frac{\eta}{s}$ is the kinetic theory value for a classical gas of massless Boltzmann particles [39] and $c_{\pi}$ is varied between $\frac{1}{4}$ and 1 .

Decoupling from the hydrodynamic fluid is implemented by following the same procedure as described in Ref. [3]. We use the AZHYDRO algorithm [48] to find the freeze-out surface at constant decoupling temperature $T_{\mathrm{dec}}=130 \mathrm{MeV}$ and calculate the final hadron spectra from the Cooper-Frye integral over this surface [49], with a distribution function that accounts for the remaining small deviations from local thermal equilibrium along that surface [3,6]. Resonance decays are neglected, and only the elliptic flow of directly emitted pions is shown. To estimate the total charged hadron multiplicity, we take the directly emitted positive pions, multiply by 1.5 to roughly account for multiplication by resonance decays at $T_{\text {dec }}$, then multiply by another factor $2 \times 1.2=2.4$ to account for the negatives and roughly $20 \%$ of final charged hadrons that are not pions. A proper calculation of the resonance decay chain is computationally expensive and, for a systematic study like the one presented here that requires hundreds of runs of VISH2+1, beyond our presently available resources.

Figure 1 shows the three equations of state (EOSs) explored in the present study. EOS I describes a noninteracting gas of massless particles, $e=p / 3$. EOS Q is a frequently employed equation of state [26] that matches a noninteracting quarkgluon gas above $T_{c}$ in a first-order transition (Maxwell construction) to a realistic hadron resonance gas (HRG) in chemical equilibrium below $T_{c}$, using a bag parameter $B$ to adjust $T_{c}$ to $T_{c}=164 \mathrm{MeV}$. SM-EOS $\mathrm{Q}$ is a slightly

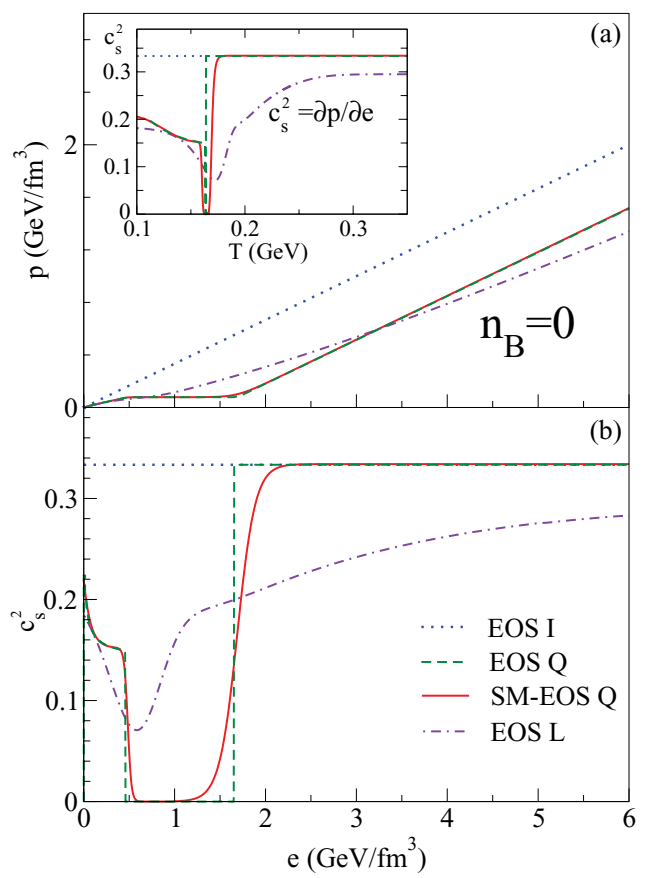

FIG. 1. (Color online) Equations of state. (a) Pressure $p$ as a function of energy density $e$ and (in the inset) the squared speed of sound $c_{s}^{2}=\frac{\partial p}{\partial e}$ as a function of temperature $T$. (b) $c_{s}^{2}$ as a function of energy density $e$. smoothed version of EOS Q, see Ref. [3] for details. Since the discontinuities in the function $c_{s}^{2}(e)$ for EOS Q cause numerical problems in VISH2+1 due to large velocity gradients near the interfaces between the QGP, mixed phase, and HRG, we here use SM-EOS Q.

EOS L matches the same hadron resonance gas below $T_{c}$ smoothly in a rapid cross-over transition to lattice QCD data [50] above $T_{c}$. For the fit, the lattice data were plotted in the form $p(e)$, interpolated, and then smoothly joined to the $p(e)$ curve of the HRG. As can be seen in the inset of Fig. 1(a), our procedure is not fully thermodynamically consistent and leads to a somewhat different temperature dependence of $c_{s}^{2}$ below $T_{c}$ than for EOS Q and SM-EOS Q. Since this only affects the flow dynamics below our decoupling temperature of $T_{\mathrm{dec}}=$ $130 \mathrm{MeV}$, we have not put any effort into correcting this. For future comparisons of viscous hydrodynamic calculations with experimental data, the chemical equilibrium hadron resonance gas below $T_{c}$ employed here must be replaced by a chemically nonequilibrated hadron gas whose particle ratios are frozen in at the chemical decoupling temperature $T_{\text {chem }} \approx T_{c}$; this has well-known consequences for the final hadron spectra and elliptic flow which cannot be neglected [51]. We postpone this, together with a more careful and thermodynamically fully consistent matching to the lattice QCD data, to a future study. We note, however, that the EOS L shown in Fig. 1 is quite similar to the "EOS qp" studied in Ref. [52].

\section{EVOLUTION OF MOMENTUM ANISOTROPIES: SIMPLIFIED VS FULL I-S EQUATIONS}

As previously observed, the results of Refs. [1,3] for the differential elliptic flow $v_{2}\left(p_{T}\right)$, although both based on the Israel-Stewart second-order formalism, seemed to disagree, that is, our work [3] shows much stronger viscous suppression of $v_{2}$ than that of Romatschke and Romatschke [1]. The resolution of this discrepancy was made difficult by the fact that the two groups not only used different versions of the Israel-Stewart equation (3) as described in Sec. II, but also different initial conditions, equations of state, and system sizes $(\mathrm{Cu}+\mathrm{Cu}$ [3] vs $\mathrm{Au}+\mathrm{Au}$ [1]). In Ref. [3] we noted in a footnote that the main reason for the observed differences seemed to be the different I-S equations used by the two groups. As we will see, this is only part of the story. In this section, we explore this question further and lead it to a complete resolution.

Figure 2 shows the temporal evolution of the total momentum anisotropy $\varepsilon_{p}=\frac{\left\langle T^{x x}-T^{y y}\right\rangle}{\left\langle T^{x x}+T^{y y}\right\rangle}$ averaged over the transverse plane $^{1}$ for two collision systems $(\mathrm{Cu}+\mathrm{Cu}$ and $\mathrm{Au}+\mathrm{Au})$ at $b=7 \mathrm{fm}$ and three equations of state [EOS I (top), SM-EOS Q (middle), and EOS L (bottom)]. The upper (blue) dashed lines

\footnotetext{
${ }^{1}$ Note that $\varepsilon_{p}$ as defined here includes the effects from both flow velocity and shear pressure anisotropies [3]. In Ref. [3] we denoted it by $\varepsilon_{p}^{\prime}$ to distinguish it from the flow-induced momentum anisotropy $\frac{\left\langle T_{0}^{x x}-T_{0}^{y y}\right\rangle}{\left\langle T_{0}^{x x}+T_{0}^{y y}\right\rangle}$, which is based only on the ideal fluid part of the energy momentum tensor and neglects anisotropies in the local fluid rest frame caused by the shear pressure tensor $\pi^{m n}$. In the present work, we drop the prime for convenience.
} 

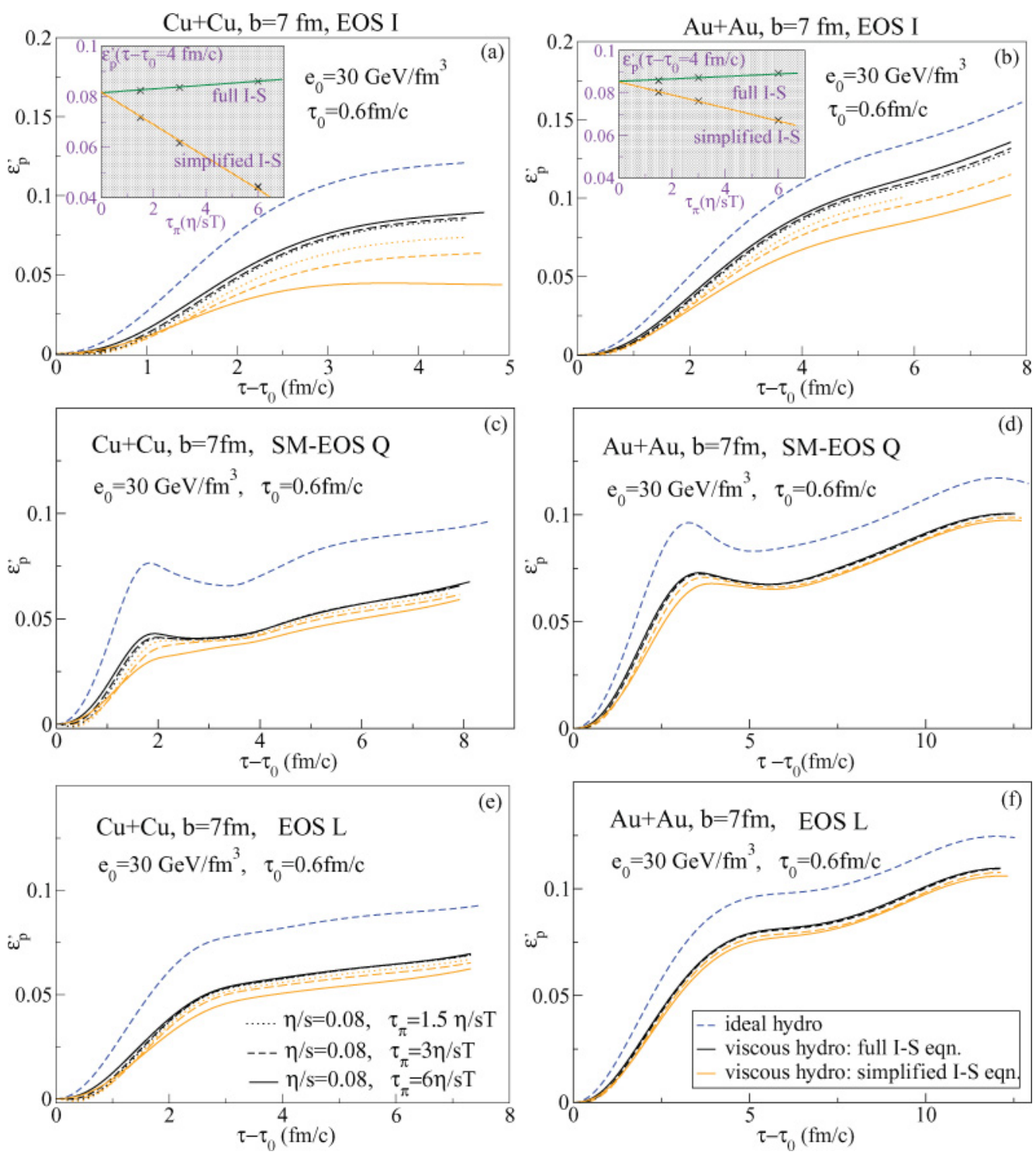

FIG. 2. (Color online) Time evolution of the total momentum anisotropy $\varepsilon_{p}^{\prime}$ for two collision systems $(\mathrm{Cu}+\mathrm{Cu}, \mathrm{Au}+\mathrm{Au})$, three $\mathrm{EOSs}$, and three values of the kinetic relaxation time $\tau_{\pi}$. The insets in (a) and (b) show the $\tau_{\pi}$ dependence of the momentum anisotropy $\varepsilon_{p}$ at fixed time $\tau-\tau_{0}=4 \mathrm{fm} / c$. See text for discussion.

indicate the result from ideal fluid dynamics, the black and gray (orange) lines below show viscous fluid dynamical results. The black lines show solutions of the full I-S equations, the gray (orange) ones for the simplified I-S approach; in each case, several values of the kinetic relaxation time $\tau_{\pi}$ are explored. Note that our full I-S equation (3) does not use the identity (4) used in Ref. [1], which strictly holds only for conformal fluids (i.e., for the case of EOS I in Fig. 2). We have, however, tested the two expressions on the left and right of Eq. (4) against each other also for the other two equations of state (SM-EOS Q and EOS L) which are not conformally invariant, and we found no discernible differences. Only for a very long relaxation time $\tau_{\pi}=12 \eta / s T$ (not shown in Fig. 2) did we see for EOS $L$ a difference larger than the line width, with our result for $\varepsilon_{p}$ lying slightly above the one obtained with the conformal approximation (4).
Comparison of the black and gray (orange) lines in Fig. 2 shows that the sensitivity of the momentum anisotropy $\varepsilon_{p}$ to the relaxation time $\tau_{\pi}$ is significantly larger for the simplified I-S equations (gray or orange) than for the full I-S equations, and that the $\tau_{\pi}$ dependence of $\varepsilon_{p}$ even has the opposite sign for the two sets of equations. With the full I-S equations, $\varepsilon_{p}$ moves slowly toward the ideal fluid limit as $\tau_{\pi}$ increases, whereas with the simplified I-S equations $\varepsilon_{p}$ moves away from the ideal fluid limit, at a more rapid rate, resulting in a larger viscous suppression of the momentum anisotropy. In the limit $\tau_{\pi} \rightarrow 0$, both formulations approach the same Navier-Stokes limit. The difference between the full and simplified I-S equations is largest for EOS I, which is the stiffest of the three studied equations of state, causing the most rapid expansion of the fireball. For this EOS, the simplified I-S equations allow for the largest excursions of $\pi^{m n}$ away from its 


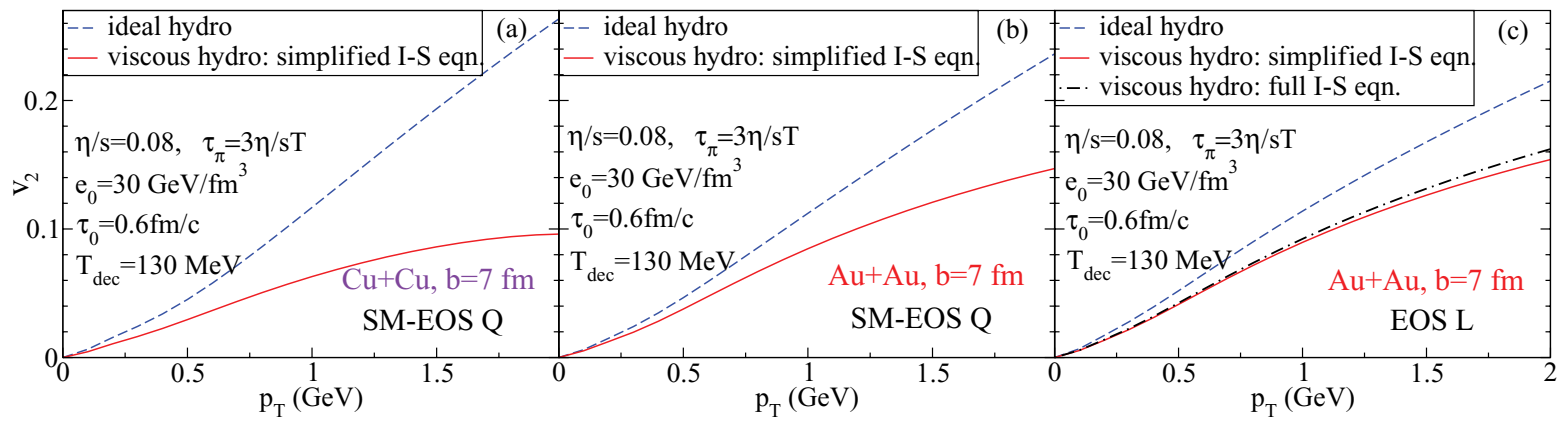

FIG. 3. (Color online) Differential elliptic flow $v_{2}\left(p_{T}\right)$ for directly emitted pions (i.e., without resonance decay contributions), comparing results for different collisions systems and EOSs, for ideal and viscous fluid dynamics, with parameters as indicated.

Navier-Stokes limit, causing a significant and strongly $\tau_{\pi}$ dependent increase of all viscous effects, including the suppression of the momentum anisotropy (Fig. 2) and elliptic flow (see Fig. 4 below) and the amount of viscous entropy production (see Sec. VI).

For the other two equations of state, SM-EOS Q and EOS L, the difference between full and simplified I-S dynamics is much smaller, ranging from $\sim 5 \%$ for $\mathrm{Au}+\mathrm{Au}$ to $\sim 15 \%$ for $\mathrm{Cu}+\mathrm{Cu}$ for the largest $\tau_{\pi}$ value of $6 \eta / s T$ studied here. Note that the viscous suppression of $\varepsilon_{p}$ is much stronger for the smaller $\mathrm{Cu}+\mathrm{Cu}$ collision system than for $\mathrm{Au}+\mathrm{Au}$. For SMEOS Q and EOS L (which yield rather similar results for $\varepsilon_{p}$, with differences not exceeding $\sim 10 \%$ ), the results from the full I-S equations (black lines) are almost completely independent of $\tau_{\pi}$, even for the small $\mathrm{Cu}+\mathrm{Cu}$ system.

The insets in Figs. 2(a) and 2(b) illustrate the different $\tau_{\pi}$ dependences for $\varepsilon_{p}$ in the full and simplified I-S formulations, by plotting the value of $\varepsilon_{p}$ for EOS I at a fixed time $\tau-\tau_{0}=$ $4 \mathrm{dm} / c$ as a function of $\tau_{\pi}$. One sees that for the investigated range of relaxation times, the $\tau_{\pi}$ dependence is linear, but the slope has different signs for the full and simplified I-S equations and is much smaller for the full I-S system. Even though VISH2+1 cannot be run for much smaller $\tau_{\pi}$ values, because of numerical instabilities that develop as the NavierStokes limit $\tau_{\pi}=0$ is approached, the lines corresponding to the full and the simplified I-S equations are seen to nicely extrapolate to the same Navier-Stokes point, as they should. For SM-EOS Q and EOS L, the corresponding lines may no longer be linear, because of phase transition effects, but they are still characterized by opposite slopes for the simplified and full I-S approaches, with almost vanishing slope in the full I-S case. This agrees with findings reported in Refs. [1,53].

Figure 3 shows the effects of changing the system size, EOS, and form of I-S equations on the differential elliptic flow $v_{2}\left(p_{T}\right)$ for directly emitted pions. The largest viscous suppression of elliptic flow (by almost $70 \%$ below the ideal fluid value at $p_{T}=2 \mathrm{GeV} / c$ ) is seen for the small $\mathrm{Cu}+\mathrm{Cu}$ system, evolved with SM-EOS Q and the simplified I-S equation. This is the result reported by us in Ref. [3]. The middle panel of Fig. 3 shows that this large $v_{2}$ suppression is almost cut in half by going from $\mathrm{Cu}+\mathrm{Cu}$ to $\mathrm{Au}+\mathrm{Au}$, the system studied in Ref. [1], even without modifying the EOS or the form of the I-S equation. Changing the EOS from
SM-EOS Q [3] to EOS L (which is close to the one used in Ref. [1]) reduces the viscous $v_{2}$ suppression by another quarter, from about $40 \%$ to less than $30 \%$ below the ideal fluid limit at $p_{T}=2$. Finally, replacing the simplified I-S equations used in Ref. [3] by the full I-S equations employed in Ref. [1] further reduces the suppression from about $28 \%$ below the ideal fluid to $\sim 25 \%$ at $p_{T}=2 \mathrm{GeV} / c$. This is consistent with the results obtained in Ref. [1].

We conclude that the biggest contribution to the large difference between the results reported in Refs. [3] and [1] arises from the different collisions systems studied, with much larger viscous effects seen in the smaller $\mathrm{Cu}+\mathrm{Cu}$ system than in $\mathrm{Au}+\mathrm{Au}$ collisions. The next most important sensitivity is to the EOS; for the most realistic EOS studied here, EOS L, the differences between using the full or simplified I-S equations with $\tau_{\pi}=3 \eta / s T$ are only about $10 \%$ on a relative scale, or about $3 \%$ on the absolute scale set by the elliptic flow from ideal fluid dynamics. For smaller $\tau_{\pi}$, this last difference would shrink even further.

The sensitivity to details of the EOS documented by Figs. 3(b) and 3(c) gives an idea of how well one needs to know the EOS if one wants to extract the specific shear viscosity $\eta / s$ from experimental data using viscous hydrodynamics. One might argue that the difference between a first-order phase transition implemented through SM-EOS Q and a smooth crossover as in EOS L should be sufficiently extreme to cover the maximal theoretical uncertainty. In this case, Fig. 3 tells us that the maximal theoretical uncertainty on the viscous suppression of $v_{2}$ (and therefore on $\eta / s$ ) should be about $25-30 \%$. This should be compared with the theoretical error introduced by our present uncertainty of the initial spatial source eccentricity $\varepsilon: \varepsilon$ differs by about $30 \%$ between initializations based on the Glauber and color glass condensate (CGC) models [19,53-55], resulting in a 30\% uncertainty of the total magnitude of the elliptic flow in ideal fluid dynamics. We further caution that recent discussions about the value of the critical temperature for the quark-hadron transition $[56,57]$ introduce an additional moment of uncertainty, which is perhaps not covered by the range between SM-EOS Q and EOS L studied here. Therefore, while we agree with the authors of Ref. [53] that the uncertainty about the initial source eccentricity dominates over uncertainties related to different implementations of the I-S formalism, we think that the EOS should not be discounted prematurely as a possible source of 


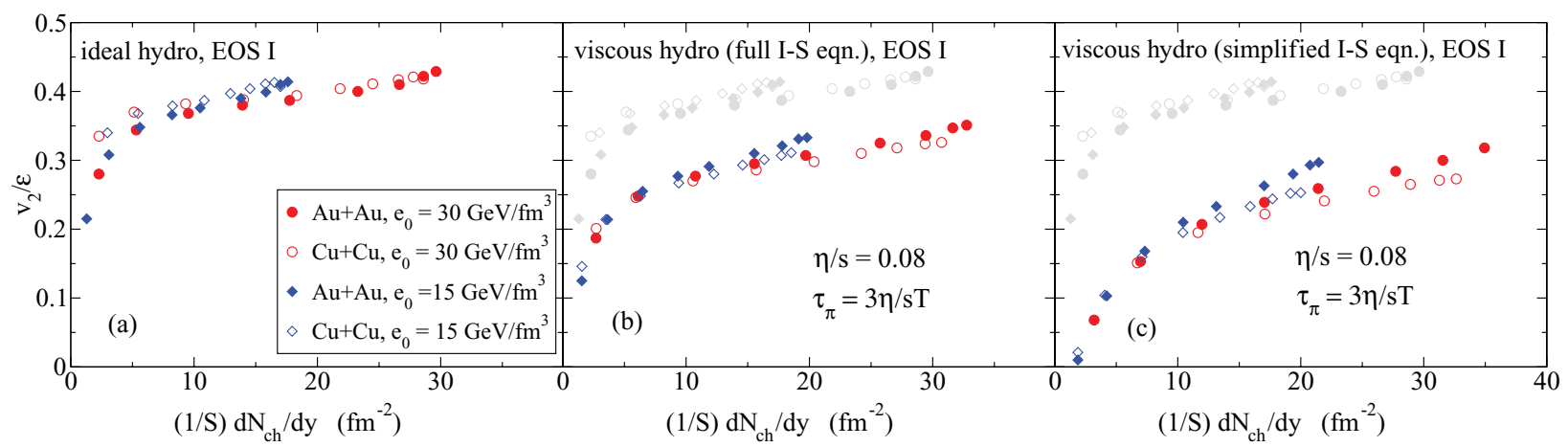

FIG. 4. (Color online) Eccentricity-scaled elliptic flow $v_{2} / \varepsilon$ as a function of charged multiplicity density $(1 / S) d N_{\text {ch }} / d y$ for a conformal fluid with EOS I. Results for $\mathrm{Cu}+\mathrm{Cu}$ and $\mathrm{Au}+\mathrm{Au}$ collisions with two different initial energy densities at a variety of impact parameters, as indicated in the legend, are superimposed. Results from ideal fluid dynamics (a) are compared with those from viscous hydrodynamics, using the full (b) and the simplified (c) Israel-Stewart equations. In all cases, approximate, but not perfect, multiplicity scaling is observed (see text for discussion). In (b) and (c), the ideal fluid results from the left panel are reproduced as light gray symbols for comparison.

significant additional theoretical uncertainty in the extraction of $\eta / s$.

\section{MULTIPLICITY SCALING OF $v_{2} / \varepsilon$ IN IDEAL AND VISCOUS HYDRODYNAMICS}

In this section, we explore the multiplicity scaling (as defined in the Introduction) of the eccentricity-scaled elliptic flow $v_{2} / \varepsilon$, comparing ideal fluid dynamics with that of near-minimally viscous fluids with specific shear viscosity $\frac{\eta}{s}=\mathcal{O}\left(\frac{1}{4 \pi}\right)$.

\section{A. EOS I: Conformal fluids with $e=3 p$}

We begin with the simple case of a conformal fluid with the equation of state $e=3 p$ (EOS I), without phase transition. In this case, the speed of sound is a constant, independent of temperature $T, c_{s}^{2}=\frac{1}{3}$. For the ideal fluid case, naive scaling arguments based on the scale invariance of the ideal fluid equations of motion would thus predict a constant $v_{2} / \varepsilon$, independent of multiplicity density $(1 / S) d N_{\mathrm{ch}} / d y$. (The nuclear overlap area $S$ is computed as $S=\pi \sqrt{\left\langle x^{2}\right\rangle\left\langle y^{2}\right\rangle}$ where $\langle\ldots\rangle$ denotes the energy density weighted average over the transverse plane.) Figure 4(a) clearly contradicts this expectation. Freeze-out at $T_{\mathrm{dec}}=130 \mathrm{MeV}$ cuts short the hydrodynamic evolution of the momentum anisotropy $\varepsilon_{p}$ before the elliptic flow has fully saturated. As Fig. 4(a) shows, this not only causes a strong suppression of $v_{2} / \varepsilon$ at low multiplicity densities, where the time between beginning of the hydrodynamic expansion and freeze-out becomes very short, but it also breaks the multiplicity scaling at high multiplicity density, albeit more weakly. At a fixed value of $(1 / S) d N_{\mathrm{ch}} / d y$, one sees larger $v_{2} / \varepsilon$ for more central collisions initiated at lower collision energies (corresponding to smaller $e_{0}$ parameters) than for more peripheral collisions between the same nuclei at higher beam energies, and also for more central $\mathrm{Cu}+\mathrm{Cu}$ collisions (with a rounder shape) than for more peripheral $\mathrm{Au}+\mathrm{Au}$ collisions (with a more deformed initial shape). We find that the larger $v_{2} / \varepsilon$ values can be traced directly to somewhat longer lifetimes of the corresponding fireballs, i.e., to the availability of more time to approach the saturation values of the momentum anisotropy and elliptic flow before reaching freeze-out. These freeze-out induced scaling violations in ideal fluid dynamics disappear at sufficiently high collision energies (i.e., large $e_{0}$ ), where the momentum anisotropy has time to fully saturate in all collision systems and at all impact parameters, before freezing out.

Figures 4(b) and 4(c) show the analogous results for a minimally viscous fluid with $\frac{\eta}{s}=\frac{1}{4 \pi}$ and kinetic relaxation time $\tau_{\pi}=\frac{3 \eta}{s T}$. Consistent with the discussion in the preceding section, the viscous suppression of the elliptic flow is seen to be stronger if the simplified I-S equation is used than for the full I-S equation. [Although not shown, the curves in Fig. 4(c) also show stronger sensitivity to the value of $\tau_{\pi}$ than those in Fig. $4(\mathrm{~b})$.] Along with the suppression of $v_{2} / \varepsilon$ by shear viscosity, we see the appearance of scale-breaking effects that increase in proportion to the overall suppression of elliptic flow: they are larger in Fig. 4(c) than in Fig. 4(b). Shear viscosity breaks the multiplicity scaling of $v_{2} / \varepsilon$ because (as shown in the preceding section) viscous effects are larger in smaller collision fireballs. Consequently, if we compare different collision systems that produce the same charged multiplicity density $(1 / S) d N_{\mathrm{ch}} / d y$, we find smaller $v_{2} / \varepsilon$ for $\mathrm{Cu}+\mathrm{Cu}$ than for $\mathrm{Au}+\mathrm{Au}$ collisions, and for peripheral $\mathrm{Au}+\mathrm{Au}$ collisions at higher collision energy than for more central $\mathrm{Au}+\mathrm{Au}$ collisions at lower collision energy.

Viscous effects also generate entropy, i.e., they increase the final charged multiplicity $d N_{\mathrm{ch}} / d y$. Comparing in the Figs. 4(b) and 4(c) the gray (shaded) symbols from ideal fluid dynamics with the colored (solid) symbols for viscous hydrodynamics, points corresponding to the same collision system and impact parameter are seen to be shifted to the right. This enhances the scaling violations: for a given collision system, impact parameter, and collision energy, viscosity decreases the eccentricity scaled elliptic flow $v_{2} / \varepsilon$, pushing the corresponding point downward in the diagram, and simultaneously increases the entropy, pushing the corresponding point horizontally to the right. The combination of these two effects separates the curves for different collision systems and energies farther in viscous hydrodynamics than in ideal fluid dynamics. 

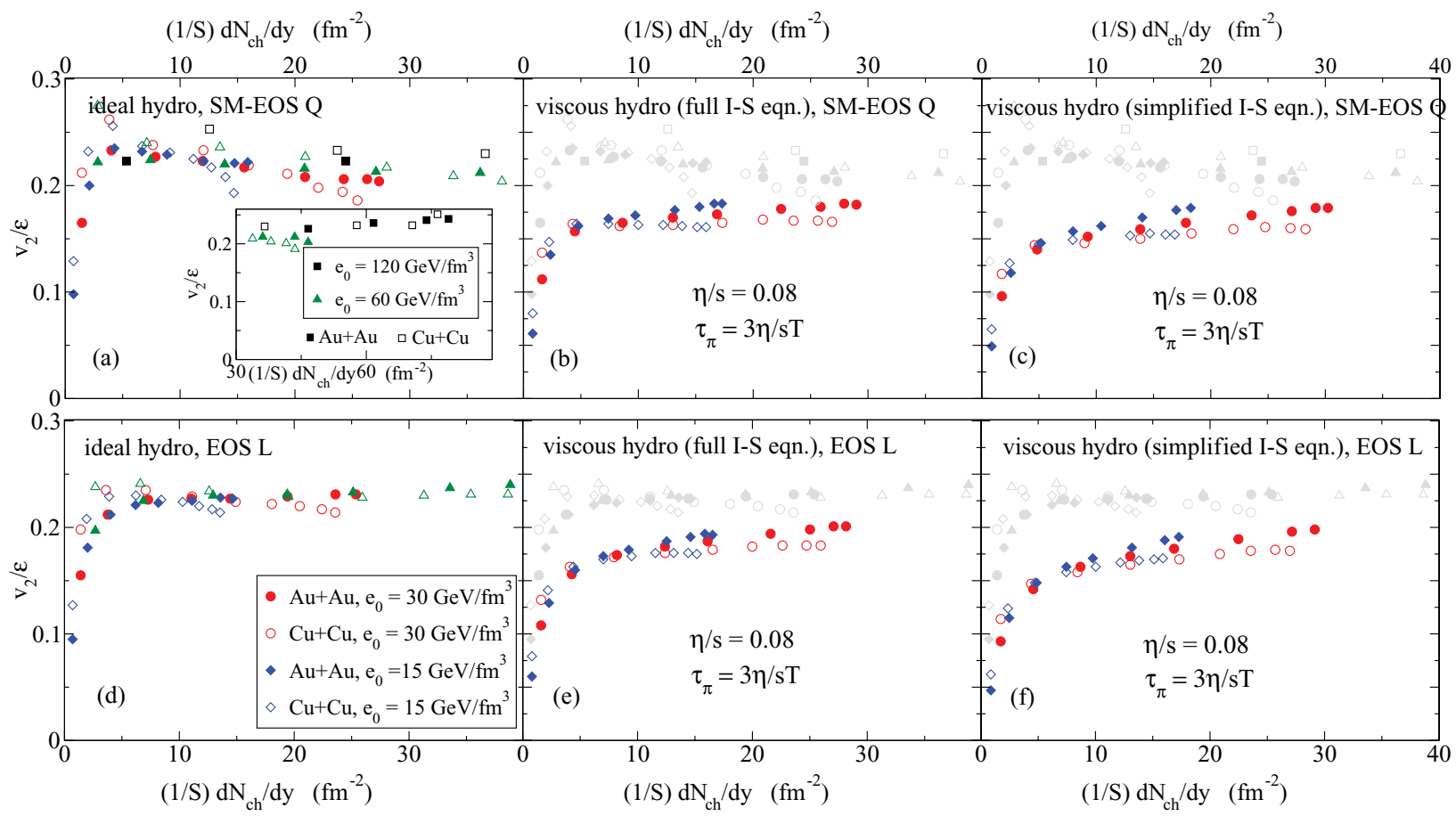

FIG. 5. (Color online) Same as Fig. 4, but for SM-EOS Q and EOS L. For the ideal fluid case in (a) and (d), an extended range of $e_{0}$ values up to $e_{0}=120 \mathrm{GeV} / \mathrm{fm}^{3}$ was studied to show that $v_{2} / \varepsilon$ eventually increases again at higher collision energies [25].

\section{B. Phase transition effects: EOS Q and EOS L}

Figure 5 shows the analogous results if the fluid evolves under the influence of an equation of state with a quark-hadron phase transition, EOS Q (top row) or EOS L (bottom row). Again, approximate multiplicity scaling of $v_{2} / \varepsilon$ is observed, but small scale-breaking effects are visible in both ideal and viscous hydrodynamics. For the equations of state with a phase transition, the scale-breaking effects are actually larger in the ideal than in the viscous case, i.e., in viscous hydrodynamics $v_{2} / \varepsilon$ shows better multiplicity scaling than in ideal fluid dynamics! We interpret the large scale-breaking effects in the ideal fluid case as a complication arising from interference between the freeze-out process and the weak acceleration of matter in the phase transition region. This interpretation is supported by a comparison between SM-EOS Q with its first-order phase transition [Fig. 5(a)] and the smooth crossover transition in EOS L [Fig. 5(d)]: for ideal fluids, the scale-breaking effects are obviously larger for SM-EOS Q than for EOS L. As already observed in Ref. [3], shear viscosity effectively smears out the phase transition and reduces its effect on the dynamics. In Fig. 5, this is clearly seen on the left side of each panel (i.e., at small values of $\frac{1}{S} \frac{d N_{\text {ch }}}{d y}$ ) where for the ideal fluid $v_{2} / \varepsilon$ shows a nonmonotonic peak structure [25] that is completely gone in the viscous case.

Comparing Figs. 4 and 5, we see much smaller differences between the full (middle panels) and simplified I-S equations (right panels) for SM-EOS Q and EOS L than for EOS I. This is consistent with our observations in Sec. IV, where the largest differences between full and simplified I-S equations was also seen for the rapidly evolving fireballs whose expansion is pushed by the very stiff EOS I.
It is interesting to observe that for ideal fluids, EOS L leads to about $10 \%$ more elliptic flow under RHIC conditions than SM-EOS Q. The reason is that in the phase transition region, EOS L is stiffer than SM-EOS Q. This plays an important role at RHIC because the softness of the EOS near $T_{C}$ inhibits the buildup of elliptic flow exactly under RHIC conditions [25]. As a corollary, we note that if RHIC elliptic flow data exhaust ideal fluid predictions made with SM-EOS Q [8], they will not exhaust ideal fluid predictions based on EOS L, thus leaving some room for shear viscous effects.

\section{Viscous suppression of $v_{2}$ : Systematics}

Even at the highest collision energies (or $e_{0}$ values) studied in Figs. 4 and 5, the slope of $v_{2} / \varepsilon$ as a function of $\frac{1}{S} \frac{d N_{\text {ch }}}{d y}$ remains positive, i.e., $v_{2} / \varepsilon$ continues to increase and evolve in direction of the asymptotic ideal fluid limit. This implies that at higher collision energies the importance of viscous effects decreases. This observation parallels the one made in Ref. [3], namely, that with increasing collision energy, the $p_{T}$ range increases over which viscous hydrodynamic predictions for the single-particle momentum spectra can be trusted. The reason is in both cases that with increasing collision energy, the time until freeze-out grows, and that (at least for constant $\eta / s$ as assumed here and in Ref. [3]) during the later stages of the expansion, shear viscous effects are small.

Figure 6 shows this more quantitatively. We plot the fractional decrease of the elliptic flow relative to its ideal fluid dynamical value, $\left(v_{2}^{\text {ideal }}-v_{2}^{\text {viscous }}\right) / v_{2}^{\text {ideal }}$, as a function of multiplicity density. Larger multiplicity densities lead to smaller viscous suppression effects. Larger viscosity results 


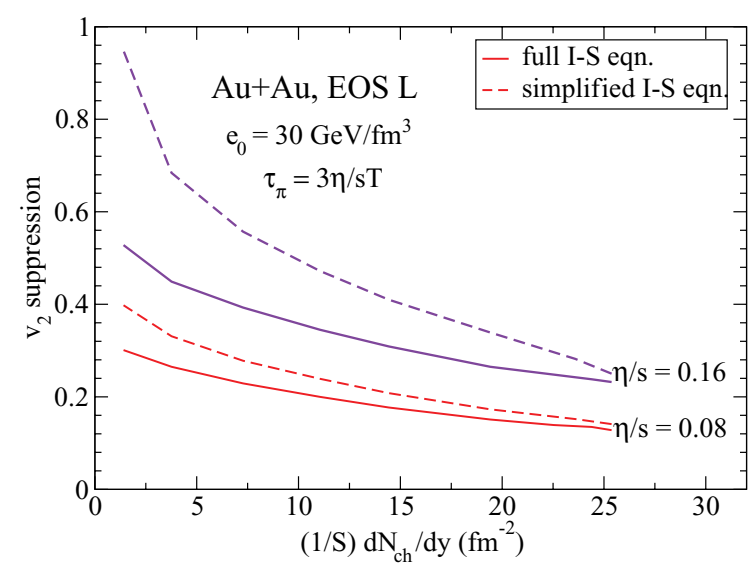

FIG. 6. (Color online) Viscous suppression of elliptic flow, $\left(v_{2}^{\text {ideal }}-v_{2}^{\text {viscous }}\right) / v_{2}^{\text {ideal }}$, as a function of $(1 / S) d N_{\mathrm{ch}} / d y$ for $\mathrm{Au}+\mathrm{Au}$ with EOS L, $\tau_{\pi}=3 \eta / s T$, and two values of $\eta / s$ as indicated, using the full and simplified I-S equations.

in stronger suppression of the elliptic flow. The suppression effects are weaker if the full I-S equations are used than in the simplified approach of Ref. [3] (which, as discussed in Sec. IV, also suffers from strong sensitivity to $\tau_{\pi}$ ). For minimal viscosity, $\eta / s=1 / 4 \pi$, the $p_{T}$-integrated elliptic flow $v_{2}$ in $\mathrm{Au}+\mathrm{Au}$ collisions at RHIC is suppressed by about $20 \%$. The suppression is larger at lower energies but will be less at the LHC energies.

\section{A look at experimental data}

Figure 7(a) shows the famous experimental plot by Voloshin [21] which provides empirical evidence for multiplicity scaling of $v_{2} / \varepsilon$. The lines labeled "HYDRO" are sketches for expectations from ideal fluid dynamics, based on the calculations presented in Ref. [25] for $v_{2}$ in $\mathrm{Au}+\mathrm{Au}$ collisions at fixed impact parameter $b=7 \mathrm{fm}$ as a function of multiplicity (parametrized by $e_{0}$ ). They should be replaced by the curves shown in the left panels of Fig. 5.
In Fig. 7(b) we present multiplicity scaling curves for $v_{2} / \varepsilon$ obtained from viscous hydrodynamics with the full I-S equations. On a superficial level, the theoretical curves show qualitative similarity with the experimental data, giving correct ball-park numbers if one assumes $\eta / s \sim 0.24 \sim 3 / 4 \pi$. Interestingly, ignoring experimental error bars, one can see evidence for small scaling violations in the experimental data whose pattern agrees with the theoretical predictions from viscous hydrodynamics (see discussion at the end of Sec. V A): the $62.5 A \mathrm{GeV} \mathrm{Au}+\mathrm{Au}$ data lie slightly above the $200 A$ $\mathrm{GeV} \mathrm{Au}+\mathrm{Au}$ points, and the $200 A \mathrm{GeV} \mathrm{Cu}+\mathrm{Cu}$ points fall slightly below the $62.5 \mathrm{~A} \mathrm{GeV} \mathrm{Au+Au} \mathrm{data.} \mathrm{Of} \mathrm{course,}$ these fine features of the experimental data are presently not statistically significant; much more precise data are needed to confirm or disprove the theoretical predictions, but upcoming high-statistic runs at RHIC should be able to deliver them.

Closer inspection of the two panels in Fig. 7 shows, however, that the theoretical scaling curves have the wrong slope: on the left side of the plot, i.e., for small multiplicity densities, the data seem to point toward larger specific shear viscosities $\frac{\eta}{s}>3 \times \frac{1}{4 \pi}$, whereas on the right side of the plot, for $\frac{1}{S} \frac{d N_{\mathrm{ch}}}{d y}>20 \mathrm{fm}^{-2}$, the experimental data require smaller shear viscosities, $\frac{\eta}{s} \lesssim(1-2) \times \frac{1}{4 \pi}$. But this is not at all unexpected: collisions represented by points in the right half of the plot correspond to high collision energies and large initial energy densities whose expanding fireballs spend the largest fraction of their life in the QGP phase. Fireballs created in collisions represented by points in the left part of the diagram have smaller initial energy densities and thus spend most of their time in the much more viscous hadronic phase [19]. A meaningful comparison between theory and experiment thus must necessarily account for the temperature dependence of $\eta / s$ and its dramatic increase during the quark-hadron phase transition [51]. This would lead to scaling curves in Fig. 7(b) with a larger slope that could better reproduce the data. What one can say already now is that the high-energy end of Fig. 7 requires very small specific shear viscosity $\eta / s$ for the QGP, of the same order as the minimal value postulated
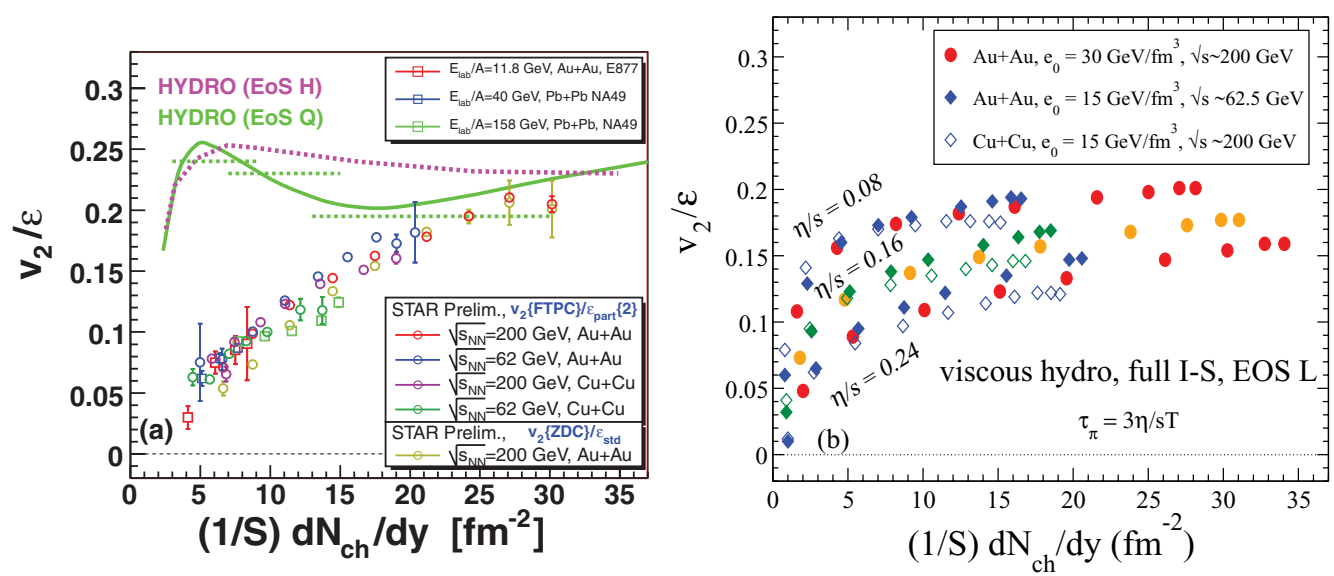

FIG. 7. (Color online) (a) Experimental observation of multiplicity scaling for $v_{2} / \varepsilon$, with data from $\mathrm{Au}+\mathrm{Au}, \mathrm{Pb}+\mathrm{Pb}, \mathrm{and} \mathrm{Cu}+\mathrm{Cu}$ collisions at RHIC, SPS, and AGS [21]. (b) Theoretical prediction of approximate multiplicity scaling from viscous hydrodynamics using the full I-S equations, for three different (constant) specific entropy values $\eta / s=0.08,0.16,0.24$. 
in Refs. [16,17] (unless the initial source eccentricity $\varepsilon$ was strongly underestimated in the experimental data).

\section{MULTIPLICITY SCALING OF ENTROPY PRODUCTION IN VISCOUS HYDRODYNAMICS}

In the absence of shock waves, ideal fluid dynamics conserves entropy. Correspondingly, the final multiplicity per unit rapidity is directly determined by the total initial entropy per unit rapidity: $\frac{d N}{d y}=$ const. $\times \tau_{0} \int d x d y s\left(x, y ; \tau_{0}\right)$. Numerical discretization of the hydrodynamic evolution equations introduces a small amount of "numerical viscosity," however, which cannot be fully avoided. To minimize numerical viscosity effects, the flux-corrected transport algorithm SHASTA used in the numerical solution of both the ideal and viscous fluid equations [3,48] employs an "antidiffusion step" involving a parameter called the "antidiffusion constant" [58]. Numerical viscosity effects are maximal if this parameter is set to zero. In all our simulations, we used 0.125 for the antidiffusion constant [58], resulting in about $0.3 \%$ entropy production by numerical viscosity in the ideal fluid case. In comparison with the $\mathcal{O}(10-15 \%)$ entropy production in a viscous fluid with minimal shear viscosity (see below), this can be neglected. ${ }^{2}$

\footnotetext{
${ }^{2}$ Effects from numerical viscosity depend on the spacing of the space-time grid used in the simulation. For the results presented in this paper, we used $\Delta x=\Delta y=0.1 \mathrm{fm}$ and $\Delta \tau=0.04 \mathrm{fm} / c$. To check the effects of numerical viscosity in the ideal fluid case, we also performed simulations with AZHYDRO where we either set the antidiffusion constant to zero or increased and decreased the grid spacings $(\Delta x, \Delta y, \Delta \tau)$ by a factor of 2 to 4 . To maximize numerical viscosity effects, we used EOS Q with a strong first-order phase transition which generates shocks and associated large velocity gradients. We found that decreasing the grid spacing by a factor of 2 has no visible effect on the average radial flow of the fluid in central $\mathrm{Au}+\mathrm{Au}$ collisions but increases the momentum anisotropy of the ideal fluid in peripheral $\mathrm{Au}+\mathrm{Au}$ collisions at $b=7 \mathrm{fm}$ by $2-3 \%$. A further reduction by another factor of 2 does not even affect the momentum anisotropy any more, indicating that numerical viscosity effects have been basically reduced to zero. If we increase the grid spacing by a factor of 2, the effects are a bit larger: the total entropy production by numerical viscosity increases by about $1 \%$, the average radial flow at $b=0$ changes by $0.5 \%$, and the momentum anisotropy experiences a relative suppression of about $5 \%$. The largest effects are seen when maximizing the numerical viscosity at fixed grid spacing by setting the antidiffusion constant to zero. In this case, the average radial flow in central $\mathrm{Au}+\mathrm{Au}$ collisions again changes only by $0.5 \%$ (which is negligible compared to the strong increase in transverse acceleration that we see in the viscous fluid), but the momentum anisotropy is reduced by about $10 \%$. In the final pion $v_{2}$ this is reflected by a $p_{T}$-dependent reduction that increases with $p_{T}$, just like effects from real shear viscosity $[1-4,6]$; the $p_{T}$-integrated pion elliptic flow is reduced by less than $4 \%$ even in this extreme case. We conclude that numerical viscosity does not increase transverse acceleration but suppresses momentum anisotropy similar to real shear viscosity. For the parameters used in this paper, and for the equations of state studied here which do not have a sharp phase transition, numerical viscosity effects on the elliptic flow do not exceed $1-2 \%$ and can thus be neglected relative to effects from real shear viscosity.
}

Similar to the second paper of Ref. [47], we compute entropy production by exploiting the proportionality of final entropy to final charged multiplicity. We compute the final multiplicity $d N_{\mathrm{ch}} / d y$ for both ideal and viscous hydrodynamics and then equate the fractional increase in $d N_{\mathrm{ch}} / d y$ with the fractional increase in $d \mathcal{S} / d y$. This ignores a small negative correction due to the viscous deviation of the distribution function on the freeze-out hypersurface from local equilibrium $[39,42]$, which slightly reduces the entropy per final observed particle in the viscous case. The real entropy production is thus slightly smaller than calculated with our prescription. However, since on the freeze-out surface the viscous pressure components are small [3], this correction should be negligible.

We checked the above procedure by also directly integrating the viscous entropy production rate $\partial \cdot s=\pi^{\mu \nu} \pi_{\mu \nu} / 2 \eta$ over the space-time volume enclosed between the initial condition Cauchy surface and the final freeze-out surface. This method results in slightly larger entropy production, the relative difference amounting to about $0.7 \%$ (or about $0.07 \%$ in the absolute value of $\Delta \mathcal{S} / \mathcal{S}_{0}$ ) for central $\mathrm{Au}+\mathrm{Au}$ collisions. Since the estimate from the final multiplicity gives a lower entropy production value even without accounting for the somewhat smaller entropy per particle in the viscous case, we conclude that entropy production due to numerical viscosity must be a bit smaller in the viscous fluid than in the ideal one. This is not unreasonable, given the observation in Ref. [3] that, compared to the ideal fluid case, the physical viscosity smooths the strong velocity gradients near the quark-hadron phase transition, thereby presumably also reducing the effects of numerical viscosity.

We note that our viscous evolution starts earlier (at $\tau_{0}=$ $0.6 \mathrm{fm} / c$ ) than that of Ref. [47] (which uses $\tau_{0}=1 \mathrm{fm} / c$ ). This earlier start results in larger entropy production fractions. As the inset in Fig. 8(b) shows, most of the entropy is produced during the early stage of the expansion. We have confirmed that the difference between Ref. [47] and the work here is quantitatively reproduced by the entropy generated during the time interval from 0.6 to $1.0 \mathrm{fm} / c$, which can be calculated to excellent approximation analytically [18] using Eq. (D3) in Ref. [3]] by assuming boost-invariant longitudinal expansion without transverse flow during this period.

Figure 8 shows the viscous entropy production $\Delta \mathcal{S}$, as a fraction of the initial entropy $\mathcal{S}_{0}$, for $\mathrm{Cu}+\mathrm{Cu}$ and $\mathrm{Au}+\mathrm{Au}$ collisions at various impact parameters and collision energies, as a function of multiplicity density. One observes approximate multiplicity scaling of the fractional entropy production, with scaling functions that depend on the equation of state and, for nonzero kinetic relaxation time, on the form of the Israel-Stewart equations used in the simulation. As with $v_{2} / \varepsilon$ we see small scale-breaking effects, but generally the produced entropy fraction shows better multiplicity scaling than elliptic flow. The scale breaking effects for the viscous entropy production rate go in the same direction as with elliptic flow insofar as, at the same value of $\frac{1}{S} \frac{d N_{\mathrm{ch}}}{d y}$, larger collision systems and more central collisions produce fractionally more entropy than smaller or more peripheral collisions, because of their longer lifetimes before freeze-out. 

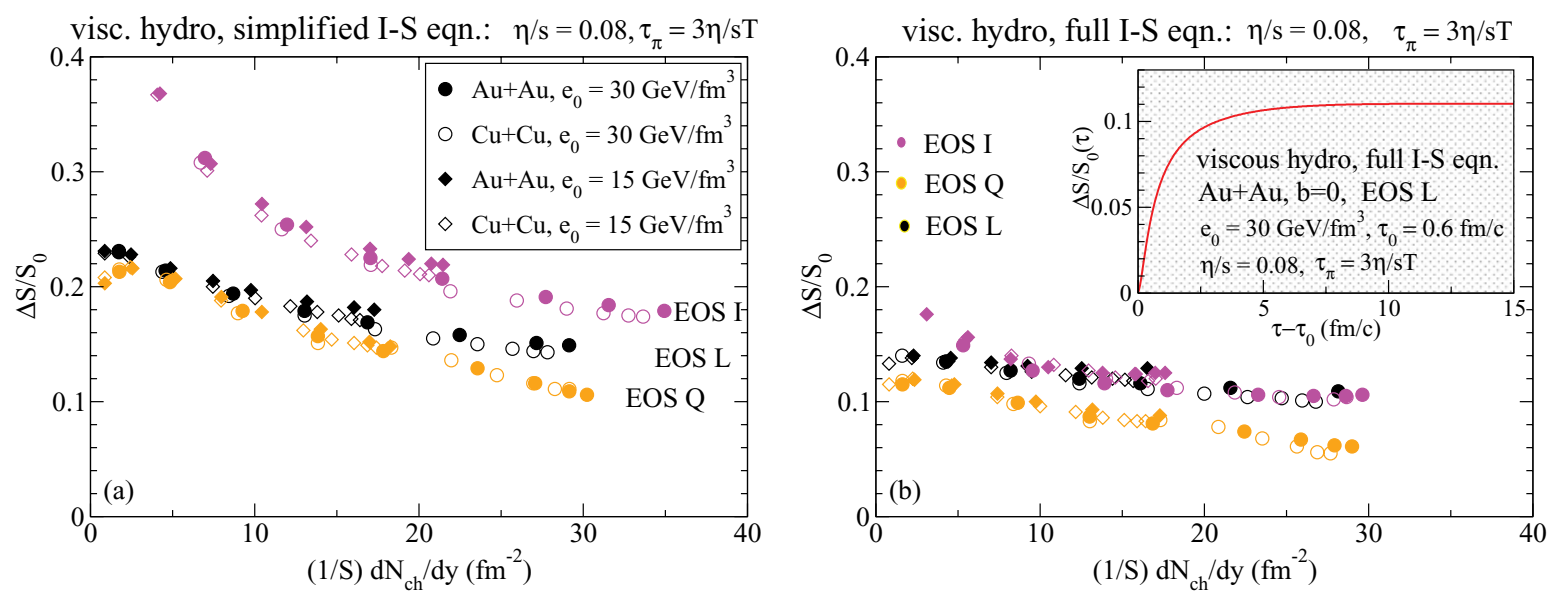

FIG. 8. (Color online) Entropy production $\Delta \mathcal{S}$, normalized by the initial entropy $\mathcal{S}_{0}$, as a function of charged multiplicity density $\frac{1}{S} \frac{d N_{\mathrm{ch}}}{d y}$. Calculations with VISH2+1 were performed for $\mathrm{Au}+\mathrm{Au}$ and $\mathrm{Cu}+\mathrm{Cu}$ collisions at various impact parameters and collision energies, using $\eta / s=0.08, \tau_{\pi}=3 \eta / s T$, and three different equations of state (EOS I, SM-EOS Q, and EOS L). (a) Simplified I-S equations. (b) Full I-S equations. The inset in (b) shows the entropy production as a function of time, for central $\mathrm{Au}+\mathrm{Au}$ collisions with parameters as indicated in the legend.

For $\tau_{\pi}=3 \eta / s T$, Fig. 8 shows that the simplified I-S equations [Fig. 8(a)] cause almost twice as much entropy production as the full I-S system! Figure 9 clarifies that when the simplified I-S equations are used, entropy production depends very sensitively on the kinetic relaxation time $\tau_{\pi}$, approaching the much smaller and almost completely $\tau_{\pi}$-independent entropy production rates of the full I-S framework in the limit $\tau_{\pi} \rightarrow 0$. The large amount of extra entropy production for nonzero $\tau_{\pi}$ in the simplified I-S approach must thus be considered as unphysical. This is important because this artificial extra entropy production (caused by unphysically large excursions of the viscous shear pressure tensor $\pi^{m n}$ away from its Navier-Stokes value $\pi^{m n}=2 \eta \sigma^{m n}$ ) manifests itself as additional charged hadron multiplicity in the observed

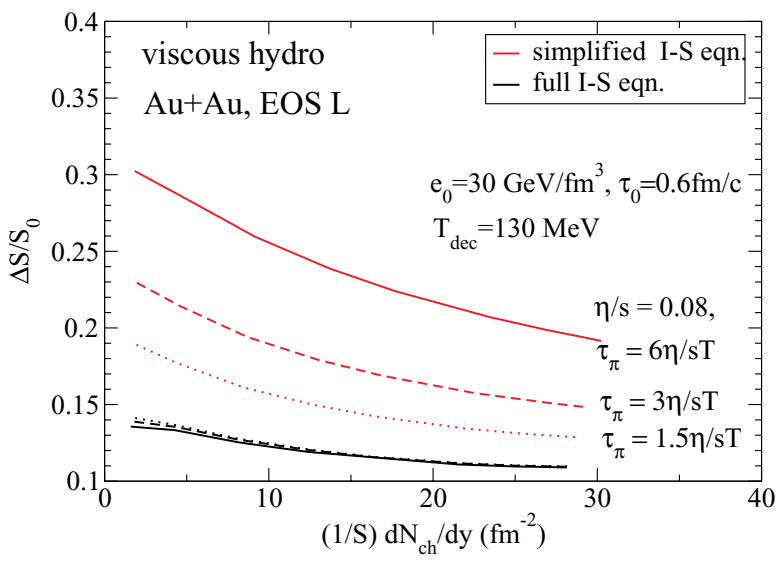

FIG. 9. (Color online) Sensitivity of the entropy production ratio $\Delta \mathcal{S} / \mathcal{S}_{0}$ shown in Fig. 8 to the kinetic relaxation time $\tau_{\pi}$, for the $\mathrm{Au}+\mathrm{Au}$ collision system with $e_{0}=30 \mathrm{GeV} / \mathrm{fm}^{3}$ (corresponding to a collision energy of $\sqrt{s} \approx 200 A \mathrm{GeV}$ ). The three red curves (upper set) are for the simplified I-S equations, the three black curves (lower set) for the full I-S equations. Solutions with the full I-S equations produce less entropy and show very little sensitivity to $\tau_{\pi}$. final state [seen as a shift of all points in Fig. 8(a) toward larger values of $\left.\frac{1}{S} \frac{d N_{\mathrm{ch}}}{d y}\right]$. Since the final multiplicity is used to normalize the initial energy density $e_{0}$, this causes a significant distortion of the initial conditions corresponding to a given set of experimental data, affecting their physical interpretation.

We conclude that using the full I-S equation is mandatory if one wants to minimize artificial effects of shear viscosity on entropy production and elliptic flow in the realistic situation of nonzero kinetic relaxation times. (We note that while the value of $\tau_{\pi}$ for the QGP created at RHIC is presently unknown, it can obviously not be zero.) From Fig. 8(b), we see that this removes most of the large difference in entropy production between the rapidly exploding EOS I fireballs and their more leisurely expanding cousins that evolve under the influence of EOS Q or EOS L. Still, even for the full I-S equations, we see $15-25 \%$ differences between the entropy production rates for EOS Q (first-order phase transition) and EOS L (rapid crossover transition). The differences are largest for the most central $\mathrm{Au}+\mathrm{Au}$ and $\mathrm{Cu}+\mathrm{Cu}$ collisions at top RHIC energies. The somewhat stiffer nature of EOS $\mathrm{L}$ near $T_{c}$ causes the fireball to expand faster and with higher acceleration, leading to larger viscous effects than for EOS Q. The differences in entropy production caused by this variation of the EOS is of similar magnitude as its effect on the viscous suppression of $v_{2} / \varepsilon$ discussed at the end of Sec. IV.

An important comment relates to the negative overall slope of the scaling curves for entropy production shown in Figs. 8 and 9: Since peripheral collisions produce relatively more entropy than central collisions, and the produced entropy is reflected in the final charged hadron multiplicity, the collision centrality dependence of hadron multiplicities is altered by viscous effects. When viscous effects are accounted for, the charged multiplicity $d N_{\mathrm{ch}} / d y$ will rise more slowly as a function of the number of participant nucleons $N_{\text {part }}$ than for an ideal fluid with the same set of initial conditions. In a Glauber model parametrization of the initial conditions [8], this tempering effect will have to be compensated for by increasing 
the "hard" component in the initial entropy production, i.e., the component that scales with the density of binary collisions and is thus responsible for the nonlinear increase of $d N_{\mathrm{ch}} / d y$ with $N_{\text {part }}$. In the color glass condensate approach [59], this nonlinear rise is controlled by the centrality dependence of the saturation momentum scale $Q_{s}$, with no free parameters to tune. It remains to be seen whether the success of the CGC model in describing the centrality dependence of $d N_{\mathrm{ch}} / d y$ [60] survives the inclusion of entropy (or multiplicity) producing effects resulting from shear viscosity during the evolution from the initial CGC to the final observed state.

\section{CONCLUDING REMARKS}

The main motivation for the work presented in this paper was provided by the experimentally observed multiplicity scaling of the elliptic flow, shown in Fig. 7(a), and its deviation at low multiplicities from ideal fluid dynamical predictions. We saw that many of the observed features are qualitatively consistent with viscous hydrodynamic calculations as presented in this paper and that the same calculations also predict approximate multiplicity scaling for viscous entropy production. Our studies revealed, however, that even for ideal fluid dynamics the multiplicity scaling of the elliptic flow is not perfect, with small scaling violations introduced by the freezeout process which cuts the evolution of elliptic flow short. Even at RHIC energies, where the elliptic flow almost saturates before freeze-out, kinetic decoupling truncates the momentum anisotropy at values slightly below their asymptotic saturation value, and the deviations depend on the size of the colliding nuclei and the deformation of the fireball created in the collision through the time available for building elliptic flow before freeze-out.

Shear viscosity strongly suppresses the buildup of momentum anisotropy and elliptic flow, especially for low multiplicity densities, i.e., at large impact parameters, low collision energies, or for small sizes of the colliding nuclei. This changes the slope of the multiplicity scaling curve for $v_{2} / \varepsilon$ but preserves, to good approximation, its general scaling with $\frac{1}{S} \frac{d N_{\mathrm{ch}}}{d y}$. Violations of multiplicity scaling for $v_{2} / \varepsilon$ are somewhat larger for the viscous expansion than for the ideal fluid (especially with EOS I), but they remain small enough to be consistent, within statistical errors, with the experimental observation of approximate scaling. The slope of the approximate scaling curve and the spread around this curve caused by scaling violation increase with the value of the specific shear viscosity $\eta / s$ and can thus be used to constrain it.

Specifically, the observed scaling violations have the following features: at fixed multiplicity density $\frac{1}{S} \frac{d N_{\mathrm{ch}}}{d y}$, viscous hydrodynamics predicts slightly larger elliptic flow $v_{2} / \varepsilon$ for larger collision systems or more central collisions than for smaller nuclei colliding at similar energy or more peripheral collisions between similar-size nuclei colliding at higher energy. Larger $v_{2} / \varepsilon$ values are associated with longer lifetimes of the corresponding fireballs before freeze-out and thus also with larger relative entropy production. This correlates the scaling violations for $v_{2} / \varepsilon$ observed in Figs. 5 and 7 with those for the relative entropy production $\Delta \mathcal{S} / \mathcal{S}_{0}$ seen in Fig. 8. The pattern of the predicted scaling violations shows qualitative agreement with experiment, although higher quality data are required to render this agreement statistically robust and quantitative.

For a fixed (i.e., temperature-independent) ratio $\eta / s$, the slope of the multiplicity scaling curve for $v_{2} / \varepsilon$ does not agree with experiment-the curves predicted by viscous hydrodynamics are too flat. The slope can be increased by allowing $\eta / s$ to increase at lower temperatures: for small multiplicity densities (very peripheral collisions or low collision energies), the data seem to require $\frac{\eta}{s}>3 \times \frac{1}{4 \pi}$, whereas at large multiplicity densities they appear to constrain the specific shear viscosity to values of $\frac{\eta}{s} \lesssim(1-2) \times \frac{1}{4 \pi}$. While this is qualitatively consistent with the idea that in high-multiplicity events the dynamics is dominated by the QGP phase (whose viscosity would thus have to be small, of order $1 / 4 \pi$ ) whereas low-multiplicity events are predominantly controlled by hadron gas dynamics (which is highly viscous [19]), much additional work is needed to turn this observation into quantitative constraints for the function $\frac{\eta}{s}(T)$.

The present study also resolves questions that arose from several recent publications of viscous hydrodynamic calculations which seemed to yield different results. We explored the effects of using different implementations of Israel-Stewart second-order theory for causal relativistic viscous hydrodynamics, by comparing the "simplified Israel-Stewart equations" previously used by us [3] with the "full Israel-Stewart equations" implemented by Romatschke and Romatschke [1]. For the simplified approach, we found a strong sensitivity of physical observables on the presently unknown kinetic relaxation time $\tau_{\pi}$ for the viscous shear pressure tensor $\pi^{m n}$, in contrast to a much weaker and basically negligible $\tau_{\pi}$ dependence in the full approach. For nonzero $\tau_{\pi}$ the simplified I-S equations allow for large excursions of $\pi^{m n}$ away from its Navier-Stokes limit $\pi^{m n}=2 \eta \sigma^{m n}$. These excursions are artificial and disappear in the Navier-Stokes limit $\tau_{\pi} \rightarrow 0$ which cannot, however, be stably simulated numerically. They cause large viscous suppression effects for the elliptic flow and large amounts of extra entropy production (i.e., extra final hadron multiplicity). From our study we conclude that the simplified I-S approach should be avoided, and that a reliable extraction of $\eta / s$ from experimental data mandates the use of the full Israel-Stewart equations [1,53]. (It is, however, permissible to use the conformal fluid approximation $[1,35]$ for the full I-S equations even if the fluid's EOS is not conformally invariant, since the differences were found to be negligible.)

In comparing our previous work [3] with that of others, we also identified other factors that significantly influence the creation of elliptic flow and thus help to account for the observed differences. For a realistic equation of state that implements a quark-hadron transition (here SM-EOS Q and EOS L), it turns out that a much more important effect than using the correct version of Israel-Stewart theory is the size of the colliding nuclei. At RHIC energies and for a realistic EOS, the viscous suppression effects for $v_{2} / \varepsilon$ in $\mathrm{Cu}+\mathrm{Cu}$ collisions are almost twice as large as for the larger $\mathrm{Au}+\mathrm{Au}$ collision system. Nonnegligible differences in the amount of viscous $v_{2}$ suppression arise also from details in the EOS, with a 
smooth crossover as implemented in EOS L giving 25-30\% less suppression than a first-order transition as in SM-EOS Q. Compared to system size effects and EOS uncertainties, the differences between simplified and full I-S theory are relatively small, affecting the viscous $v_{2}$ suppression at the $10 \%$ level relative to each other. (The quoted percentages are for a fluid with minimal viscosity $\eta / s=1 / 4 \pi$ and may be larger for higher viscosity.) The largest uncertainty, in any case, is contributed by our present lack of knowledge of the initial source eccentricity, which contributes a theoretical error band of up to $30 \%$ on an absolute scale for $v_{2}$ [19,53-55].

We finally comment that the multiplicity dependence of viscous entropy production predicted by viscous hydrody- namics (see Fig. 8) will modify the centrality dependence of charged hadron production. This issue will be studied more quantitatively in a forthcoming paper.

\section{ACKNOWLEDGMENTS}

We thank K. Dusling, E. Frodermann, P. Huovinen, M. Lisa, P. Romatschke, and D. Teaney for fruitful discussions and useful comments on the manuscript. This work was supported by the U.S. Department of Energy under Contract DE-FG02$01 E R 41190$.
[1] P. Romatschke and U. Romatschke, Phys. Rev. Lett. 99, 172301 (2007).

[2] A. K. Chaudhuri, arXiv:0704.0134 [nucl-th]; arXiv:0708.1252 [nucl-th]; arXiv:0801.3180 [nucl-th]; and arXiv:0803.0643 [nucl-th].

[3] H. Song and U. Heinz, Phys. Lett. B658, 279 (2008); Phys. Rev. C 77, 064901 (2008).

[4] K. Dusling and D. Teaney, Phys. Rev. C 77, 034905 (2008).

[5] U. Heinz and S. M. H. Wong, Phys. Rev. C 66, 014907 (2002).

[6] D. Teaney, Phys. Rev. C 68, 034913 (2003).

[7] The experimental situation is summarized in I. Arsene et al. (BRAHMS Collaboration), Nucl. Phys. A757, 1 (2005); B. B. Back et al. (PHOBOS Collaboration), ibid. A757, 28 (2005); J. Adams et al. (STAR Collaboration), ibid. A757, 102 (2005); K. Adcox et al. (PHENIX Collaboration), ibid. A757, 184 (2005).

[8] Recent theoretical reviews include P. Huovinen, in Quark Gluon Plasma 3, edited by R. C. Hwa and X. N. Wang (World Scientific, Singapore, 2004), p. 600; P. F. Kolb and U. Heinz, ibid., p. 634; P. Huovinen and P. V. Ruuskanen, Annu. Rev. Nucl. Part. Sci. 56, 163 (2006).

[9] U. Heinz, J. Phys. G: Nucl. Part. Phys. 31, S717 (2005).

[10] R. A. Lacey et al., Phys. Rev. Lett. 98, 092301 (2007).

[11] U. Heinz and P. F. Kolb, Nucl. Phys. A702, 269 (2002).

[12] U. Heinz, AIP Conf. Proc. 739, 163 (2005).

[13] M. Gyulassy, in Structure and Dynamics of Elementary Matter, edited by W. Greiner et al., NATO Science Series II: Mathematics, Physics and Chemistry, Vol. 166 (Kluwer Academic, Dordrecht, 2004), p. 159 [arXiv: nucl-th/0403032].

[14] M. Gyulassy and L. McLerran, Nucl. Phys. A750, 30 (2005).

[15] E. V. Shuryak, Nucl. Phys. A750, 64 (2005).

[16] G. Policastro, D. T. Son, and A. O. Starinets, Phys. Rev. Lett. 87, 081601 (2001); J. High Energy Phys. 09 (2002) 043.

[17] P. K. Kovtun, D. T. Son, and A. O. Starinets, Phys. Rev. Lett. 94, 111601 (2005).

[18] P. Danielewicz and M. Gyulassy, Phys. Rev. D 31, 53 (1985).

[19] T. Hirano, U. Heinz, D. Kharzeev, R. Lacey, and Y. Nara, Phys. Lett. B636, 299 (2006); J. Phys. G 34, S879 (2007); Phys. Rev. C 77, 044909 (2008).

[20] C. Alt et al. (NA49 Collaboration), Phys. Rev. C 68, 034903 (2003).

[21] S. A. Voloshin, J. Phys. G 34, S883 (2007); AIP Conf. Proc. 870, 691 (2006).

[22] R. C. Hwa and K. Kajantie, Phys. Rev. D 32, 1109 (1985).
[23] J. Y. Ollitrault, Phys. Rev. D 46, 229 (1992).

[24] R. S. Bhalerao, J. P. Blaizot, N. Borghini, and J. Y. Ollitrault, Phys. Lett. B627, 49 (2005).

[25] P. F. Kolb, J. Sollfrank, and U. Heinz, Phys. Lett. B459, 667 (1999); Phys. Rev. C 62, 054909 (2000).

[26] G. Torrieri, Phys. Rev. C 76, 024903 (2007).

[27] H. Heiselberg and A. M. Levy, Phys. Rev. C 59, 2716 (1999).

[28] S. A. Voloshin and A. M. Poskanzer, Phys. Lett. B474, 27 (2000).

[29] H. J. Drescher, A. Dumitru, C. Gombeaud, and J. Y. Ollitrault, Phys. Rev. C 76, 024905 (2007).

[30] K. Paech and S. Pratt, Phys. Rev. C 74, 014901 (2006).

[31] D. Kharzeev and K. Tuchin, arXiv:0705.4280 [hep-ph]; F. Karsch, D. Kharzeev, and K. Tuchin, Phys. Lett. B663, 217 (2008).

[32] H. B. Meyer, Phys. Rev. Lett. 100, 162001 (2008).

[33] U. Heinz, H. Song, and A. K. Chaudhuri, Phys. Rev. C 73, 034904 (2006).

[34] A. Muronga and D. H. Rischke, arXiv:nucl-th/0407114.

[35] R. Baier, P. Romatschke, D. T. Son, A. O. Starinets, and M. A. Stephanov, J. High Energy Phys. 04 (2008) 100.

[36] S. Bhattacharyya, V. E. Hubeny, S. Minwalla, and M. Rangamani, J. High Energy Phys. 02 (2008) 045.

[37] M. Natsuume and T. Okamura, Phys. Rev. D 77, 066014 (2008); M. Natsuume and T. Okamura, arXiv:0801.1797 [hep-th].

[38] R. Loganayagam, J. High Energy Phys. 05 (2008) 087.

[39] W. Israel, Ann. Phys. (NY) 100, 310 (1976); W. Israel and J. M. Stewart, Phys. Lett. A58, 213 (1976); W. Israel and J. M. Stewart, Ann. Phys. (NY) 118, 341 (1979).

[40] A. Muronga, Phys. Rev. Lett. 88, 062302 (2002); 89, 159901(E) (2002)].

[41] A. Muronga, Phys. Rev. C 69, 034903 (2004).

[42] A. Muronga, Phys. Rev. C 76, 014909 (2007).

[43] R. Baier, P. Romatschke, and U. A. Wiedemann, Phys. Rev. C 73, 064903 (2006).

[44] I. Müller, Z. Phys. 198, 329 (1967).

[45] M. Grmela and H. C. Öttinger, Phys. Rev. E 56, 6620 (1997); H. C. Öttinger and M. Grmela, ibid. 56, 6633 (1997); H. C. Öttinger, ibid. 57, 1416 (1998).

[46] A. K. Chaudhuri and U. Heinz, J. Phys. Conf. Ser. 50, 251 (2006).

[47] R. Baier and P. Romatschke, Eur. Phys. J. C 51, 677 (2007); P. Romatschke, ibid. 52, 203 (2007).

[48] The (2+1)-d ideal fluid code AZHYDRO can be downloaded from http://nt3.phys.columbia.edu/people/molnard/OSCAR/. 
[49] F. Cooper and G. Frye, Phys. Rev. D 10, 186 (1974).

[50] S. D. Katz, Nucl. Phys. A774, 159 (2006).

[51] T. Hirano and M. Gyulassy, Nucl. Phys. A769, 71 (2006).

[52] P. Huovinen, Nucl. Phys. A761, 296 (2005).

[53] M. Luzum and P. Romatschke, arXiv:0804.4015 [nucl-th] (Phys. Rev. C, in press).

[54] A. Adil, H. J. Drescher, A. Dumitru, A. Hayashigaki, and Y. Nara, Phys. Rev. C 74, 044905 (2006); H. J. Drescher and Y. Nara, ibid. 75, 034905 (2007).

[55] T. Lappi and R. Venugopalan, Phys. Rev. C 74, 054905 (2006).
[56] Y. Aoki, Z. Fodor, S. D. Katz, and K. K. Szabo, Phys. Lett. B643, 46 (2006).

[57] M. Cheng et al., Phys. Rev. D 74, 054507 (2006).

[58] H. Von Gersdorff, L. McLerran, M. Kataja, and P. V. Ruuskanen, Phys. Rev. D 34, 794 (1986).

[59] D. Kharzeev and M. Nardi, Phys. Lett. B507, 121 (2001); D. Kharzeev, E. Levin, and M. Nardi, Phys. Rev. C 71, 054903 (2005).

[60] D. Kharzeev and E. Levin, Phys. Lett. B523, 79 (2001). 